\title{
How does diurnal intermittent fasting impact sleep, daytime sleepiness, and markers of the biological clock? Current insights
}

This article was published in the following Dove Press journal:

Nature and Science of Sleep

\author{
Aljohara S Almeneessier ${ }^{1,2}$ \\ Ahmed S BaHammam' \\ 'Department of Medicine, University \\ Sleep Disorders Center, College \\ of Medicine, King Saud University, \\ Riyadh, Saudi Arabia; ${ }^{2}$ Department of \\ Family Medicine, College of Medicine, \\ King Saud University, Riyadh, Saudi \\ Arabia
}

\begin{abstract}
Mealtimes and feeding schedules may interfere with the circadian system and impact sleep. The practice of intermittent fasting (IF) in its different formats is increasing worldwide. However, most studies addressing the effect of IF on circadian rhythms, daytime sleepiness, and sleep architecture have been conducted during diurnal IF for Ramadan. In this article, we analyze the effect of diurnal IF on the circadian clock, sleep, and daytime sleepiness. In freeliving, unconstrained environments that do not control for lifestyle changes such as sleep/wake schedules, sleep duration, and light exposure, studies have demonstrated sudden and significant delays in bedtime and wake time during diurnal intermittent fasting for Ramadan. However, subsequent studies that accounted for lifestyle factors and sleep/wake patterns have reported no changes in markers of the biological clock, daytime sleepiness, or sleep parameters. Nevertheless, several researchers have demonstrated a reduction in the proportion of rapid eye movement stage sleep as the significant alteration in sleep architecture during fasting.
\end{abstract}

Keywords: mealtime, Ramadan, chronotype, sleep architecture, alertness, food, light, caloric restriction

\section{Introduction}

Fasting, mealtime, and sleep may interact and impact the circadian rhythm of various body organs and cells when food is not consumed at a suitable time relative to the timing of the circadian clock of the body. ${ }^{1,2}$ Intermittent fasting (IF), in which one voluntarily refrains from food intake for specific times, is an old tradition that is implemented in different practices by various societies around the world. ${ }^{3,4}$ Several religions practice periods of fasting in their rituals, including Islam, Christianity, Judaism, Hinduism, and Buddhism. ${ }^{5}$ Recently, IF practice has become popular, and there has been great attention to the physiological and metabolic consequences of IF among researchers. ${ }^{1}$

IF is different from caloric restriction (CR), in which caloric intake is lowered for long periods by $20 \%-40 \%$ but meal frequency is preserved. ${ }^{6}$ Several types of IF have been described in the literature, including complete fasting every other day, ${ }^{7}$ significant CR every other day, ${ }^{8}$ eating only a small amount of calories (500-700) 2 consecutive days/week, " "time-restricted feeding," where food intake is restricted to a specified time daily, ${ }^{10}$ fasting 1 or 2 days per week and allowing 5-6 days ad libitum ingestion of food, and the spiritual diurnal IF practiced during Ramadan, in which there is total abstinence from food and drink during daytime (dawn to dusk). ${ }^{11,12}$

Most of the previous studies on experimental IF have assessed its effect on metabolic and cardiovascular risks. ${ }^{1}$ Most studies addressing the impact of IF on sleep and circadian rhythm have been conducted during diurnal IF for Ramadan. Therefore, in this article,
Correspondence: Ahmed S BaHammam Department of Medicine, University Sleep Disorders Center, College of Medicine, King Saud University, Box 225503, Riyadh I I324, Saudi Arabia

Tel +966 II 4679179

Fax +966 II 4679495

Email ashammam2@gmail.com 
we address the impact of diurnal IF on some markers of the circadian clock, sleep parameters, and daytime sleepiness.

\section{IF for Ramadan}

Diurnal IF for Ramadan is distinct from other forms of experimentally studied fasting for several reasons; therefore, it needs to be treated as a separate entity. Ramadan fasting comprises diurnal IF from dawn to sunset, where participants refrain from eating and drinking during the specified period. Ramadan is a month of the Arabic (Hijri) year and hence follows the lunar system. This implies that Ramadan comes during a different season every 9 years, which could affect the duration of daytime and hence fasting duration, where daytime is longer during the summer than in the winter. ${ }^{13}$

Moreover, diurnal IF is practiced for the full month of Ramadan; this long duration may permit greater adjustment to the fasting protocol than that typically occurs in other forms of experimental IF. Furthermore, several studies have shown that Ramadan is accompanied by lifestyle changes that may influence sleep and circadian rhythms (Figure 1).

\section{Review method}

The literature search started on the May 1, 2018, with the following keywords: "sleep," "sleep pattern," "daytime sleepiness," "polysomnography," "sleep architecture," "circadian rhythm," "intermittent fasting," and "Ramadan," using PubMed (80 results), Clarivate (94 results), and Google Scholar (105 results). In addition, the reference lists of the

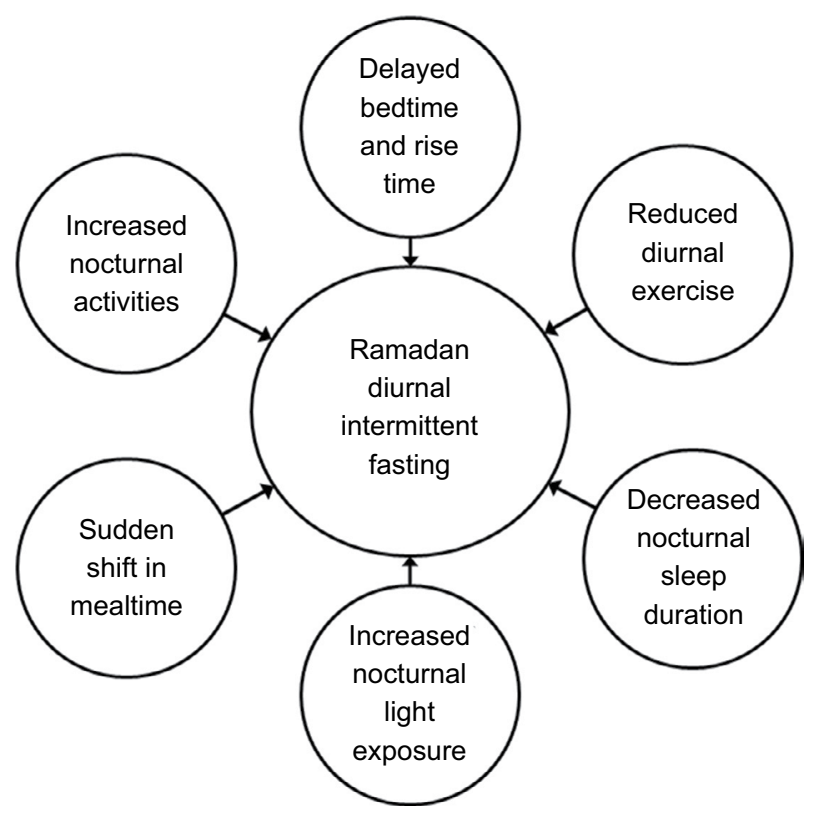

Figure I Lifestyle changes that accompany the month of Ramadan and that may affect sleep and circadian rhythms. identified articles were also searched for further references. The following inclusion criteria for human studies were used: children, men, and women were accepted as participants, and both randomized controlled trials and nonrandomized trials were accepted.

\section{Impact of diurnal IF on the circadian rhythm}

All cells of the human body have circadian clocks, which are generally classified into peripheral and central clocks based on their anatomical place. The central master clock is located in the suprachiasmatic nucleus (SCN) of the hypothalamus. On the other hand, the peripheral clocks are located in different organs and tissues. ${ }^{14}$ Both central and peripheral clocks act to preserve the circadian rhythm of different tissue physiology via controlling "tissue-specific gene expression."14

With the aim of ensuring external synchrony between the body and the surrounding environment, besides internal synchrony and correct chronological alignment between the central and peripheral clocks, the timing of the body circadian system must be calibrated repeatedly. ${ }^{14}$ The main entrainment factor (or "Zeitgeber") for the SCN is light, while peripheral circadian clocks are affected by "neurohumoral modulation." 15 Therefore, certain behaviors such as the timing of feeding and the timing of abstinence of food may affect the circadian rhythm. ${ }^{1,12,16}$ Data that address the impact of IF on the circadian rhythm are limited, and most of those data address the impact of Ramadan diurnal IF on the circadian rhythm. However, current evidence shows that high caloric consumption can lead to alterations in "clock gene expression" in the SCN and other central nuclei. ${ }^{17,18}$ In addition, animal studies have shown that temporal food restriction and enforced meal times are powerful synchronizers for body clocks in peripheral tissues, while maintaining a fixed light-dark cycle exposure. ${ }^{19}$ Moreover, it has been shown that mealtime schedules can trigger the activation of neural circadian clock genes in several species, which is assumed to be controlled by a "food-entrainable clock." 2,20

Almost all physiological systems in the body follow a circadian rhythm, which is reflected by measuring certain hormones and core body temperature. ${ }^{21}$ During Ramadan diurnal IF, 2-3 meals are taken after sunset: breakfast (a light meal) is taken at sunset, dinner is taken following night prayer in some countries ( $\sim 1-3$ hour after sunset), and a "predawn meal" (Suhur), which means that food consumption, is moved to the hours of darkness. This food intake shift partly inverts the normal circadian pattern of food intake. In theory, this 
shift in mealtime may disrupt the circadian rhythm and biological clock of fasting participants. ${ }^{12}$

\section{Subjective assessment of chronotype}

One study assessed chronotypes using an abridged version of the Horne and Ostberg questionnaire in fasting and nonfasting individuals during Ramadan diurnal IF in a "free-living environment" to define three behavioral groups: morning-type, evening-type, and neither-type. ${ }^{22,23}$ Interestingly, the study reported an increase in the evening chronotype among the performers of fasting at both the beginning and the end of Ramadan month compared with baseline. Similarly, an increase in evening chronotype was also reported in nonfasting people during Ramadan, which advocates that lifestyle changes other than fasting may influence the circadian rhythm during Ramadan. ${ }^{23}$ This result strengthens the hypothesis that lifestyle changes accompanying Ramadan may affect circadian rhythms.

\section{Effects of diurnal IF on body temperature}

Core body temperature increases during the day and decreases at night. Sleep onset is accompanied by a decrease in core body temperature, whereas an increase in core body temperature initiates the wakefulness process. ${ }^{21}$ Studies that assessed changes in core body temperature during Ramadan diurnal IF have reported conflicting results (Table 1). In general, studies that assessed the circadian rhythm during Ramadan diurnal intermittent fasting can be divided into two categories: category 1 encompasses studies that examined the changes in some markers of the circadian clock in an unconstrained "free-living environment" without controlling for confounders and lifestyle changes such as meal composition, caloric intake, total sleep time, energy expenditure, or light exposure, ${ }^{24-27}$ and category 2 encompasses studies that controlled for the above confounders. ${ }^{28-30}$

Twenty-four-hour continuous monitoring of rectal temperature in six healthy volunteers during Ramadan revealed delays in both the "bathyphase" and the "acrophase" of body temperature (ie, the times at which the calculated minimum and maximum values of body temperature occur, respectively) ${ }^{25}$ One more study reported that the circadian rhythm of body temperature was reversed during Ramadan diurnal IF, with significant decreases in oral temperature at the following times: 09:00, 11:00, 13:00, and 16:00, and considerable increases in temperature at 23:00 and 00:00 hours. ${ }^{26}$ Conversely, a third paper that assessed the oral temperature of volunteers during the first and third weeks of Ramadan at 08:00, 16:00, and 00:00 using a "high-precision" thermometer while controlling for the composition of meals and sleep duration reported no changes in body temperature. ${ }^{30}$ The above studies did not control for most of the lifestyle changes discussed earlier that may impact the circadian rhythm regardless of fasting. To assess the potential effects of lifestyle changes that accompany Ramadan, a well-designed study recruited young adults with "delayed sleep phase disorder" (evening chronotype) who used to sleep during the daytime and wake up and eat at night. ${ }^{24}$ In an unconstrained, free-living environment, the investigators use a portable armband physiological and activity sensor to measure circadian alterations in proximal skin temperature and energy expenditure before Ramadan and during the first 2 weeks of Ramadan. ${ }^{24}$ Interestingly, the study revealed a further delay in the "acrophase" of proximal skin temperature and energy expenditure denoting a shift in the circadian pattern. The findings of this study support the hypothesis that factors other than the sudden shift in mealtimes may influence sleep patterns and circadian rhythms during Ramadan. ${ }^{24}$ Table 1 summarizes the studies that have examined the circadian rhythm of body temperature during diurnal IF for Ramadan.

\section{Effects of diurnal IF on melatonin and other hormones}

Melatonin is a useful marker of circadian rhythm because it is highly precise and accurate. ${ }^{26,31,32}$ Therefore, proper measurement of melatonin level is an excellent method to examine the impact of diurnal IF on the circadian rhythm. One study assessed the blood levels of melatonin every 4 hours (not including the 02:00 time point measurement to avoid sleep disruption) before and at the end of the third week of Ramadan. ${ }^{27}$ The study revealed a reduced, delayed night peak and a flattened slope in serum melatonin concentrations during Ramadan. ${ }^{27}$ Another study, which measured the salivary melatonin levels of eight healthy volunteers (who performed diurnal IF) over a 24 hours period (08:00, 16:00, and 00:00) at three time points - 1 week before Ramadan and at the end of the first and third weeks of Ramadan - while controlling for total sleep time, revealed a statistically significant fall in melatonin concentrations at 00:00 and 16:00 during Ramadan compared to baseline levels. ${ }^{30}$ Although the study reported comparable trends in melatonin circadian pattern during Ramadan and at baseline, the slope was flatter in the former case. ${ }^{30}$ However, the above studies failed to measure melatonin late at night, neglecting the possibility of a latenight peak in melatonin concentrations.

None of the above studies accounted for potential confounders that may accompany Ramadan and affect the measured melatonin levels. In a subsequent study, 


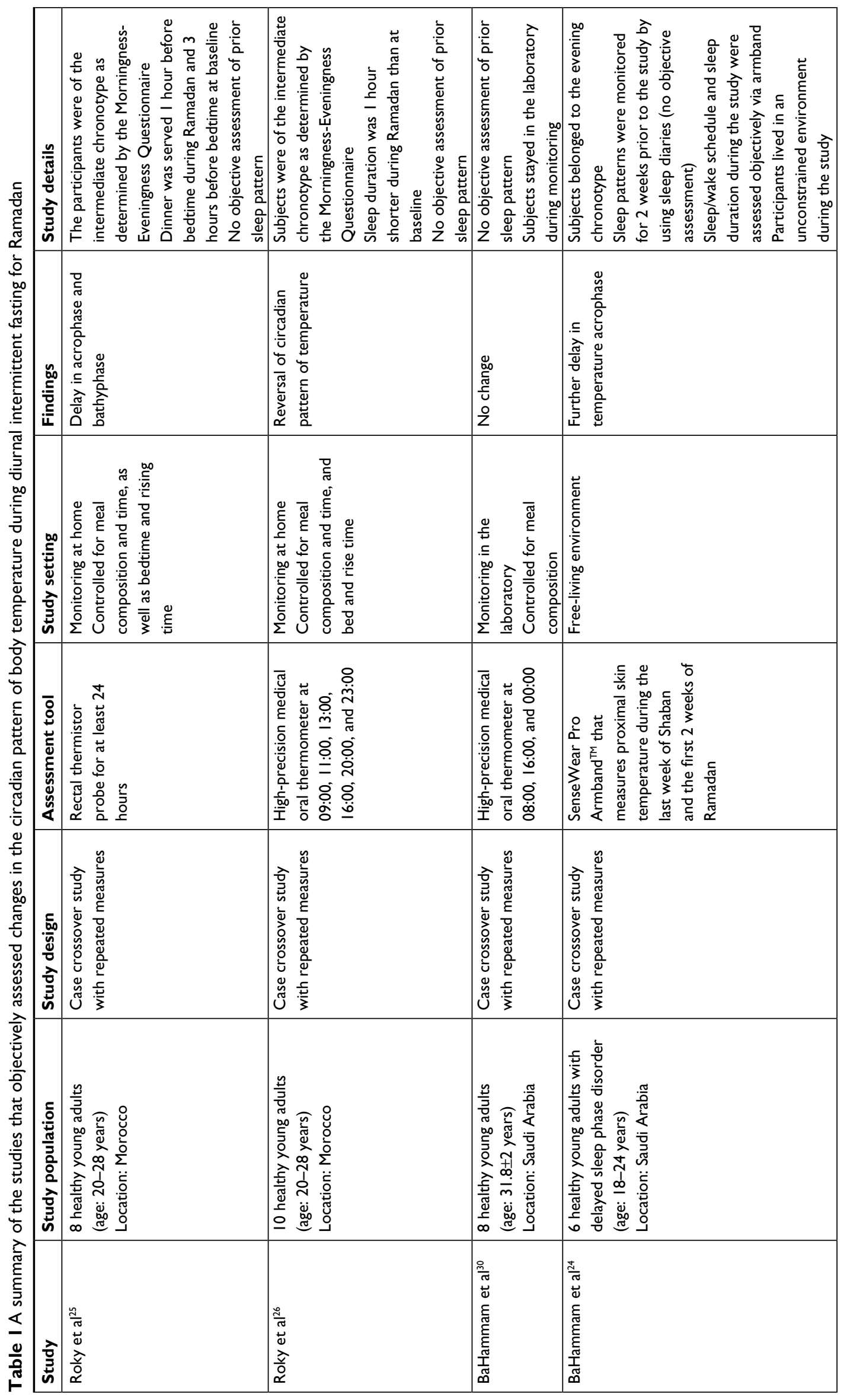


we attempted to overcome those limitations by assessing circadian profiles of serum melatonin during diurnal IF outside Ramadan (to assess the impact of diurnal IF act in the absence of the lifestyle changes associated with Ramadan) and during the second week of Ramadan while controlling for light exposure, sleep pattern, total sleep time, and meal composition. ${ }^{28}$ In eight healthy volunteers, serum melatonin was measured at five time points $(22: 00$, 02:00, 04:00, 06:00, and 11:00) three times: 4 weeks before Ramadan where volunteers were asked to perform diurnal IF (fasting outside the month of Ramadan) for 1 week, 1 week before Ramadan during which volunteers were not fasting and living a routine life, and during the second week of Ramadan while performing diurnal IF. The trough in melatonin levels occurred 11:00 during the three studied periods, which shows that diurnal IF has no significant effect on the circadian rhythm of melatonin when other confounders are accounted for. In addition, a cosinor analysis of melatonin levels showed no significant changes in the "acrophase." 28 Therefore, the previously reported changes in melatonin levels during Ramadan could be related to the lifestyle changes that accompany Ramadan. Nonetheless, more studies with larger sample sizes are required to evaluate the effects of diurnal IF on melatonin levels and circadian patterns. Table 2 summarizes the studies that have objectively addressed changes in the circadian pattern of melatonin during diurnal IF for Ramadan. ${ }^{27,28,30}$

Most studies assessing melatonin levels during diurnal IF have consistently reported a reduction in measured melatonin levels, even during short-term experimental fasting. ${ }^{30}$ Several theories have been suggested to understand the decrease in melatonin levels during fasting. The nocturnal increase in cortisol levels during Ramadan is a possible mechanism..$^{33,34}$ In addition, it has been proposed that a decrease in the glucose supply during fasting may result in a decrease in melatonin synthesis, ${ }^{35}$ as it has been shown in rats that mild hypoglycemia decreases melatonin levels, and administration of glucose improves the decrease in melatonin levels. ${ }^{36}$ However, previous studies have shown that hypoglycemia does not happen in healthy individuals during diurnal IF. ${ }^{37}$ Reduction in tryptophan had been proposed as a possible mechanism of the reduction in melatonin levels during Ramadan diurnal IF, as tryptophan is essential for melatonin synthesis. Nevertheless, this proposed mechanism is improbable because short duration diurnal IF is not likely to cause a reduction in tryptophan. ${ }^{36}$

Both leptin and ghrelin hormones are influenced by fasting, mealtime, and sleep. Hence, the abrupt shift in mealtime during diurnal IF for Ramadan may entrain the circadian rhythms of both hormones.

Two previous studies have examined the impact of diurnal IF on the circadian patterns of leptin using a cosinor analysis. The first study was conducted on free-living participants and revealed a major shift ( $\sim 5$ hours) in the peak and nadir serum leptin concentrations in the third week of Ramadan. ${ }^{38}$ However, the amplitude and 24 hours mean concentrations of leptin levels revealed no significant changes compared to the levels before Ramadan. Another study reported a significant decrease in plasma leptin concentration at 22:00; however, a cosinor analysis demonstrated no significant changes in the circadian rhythm of leptin. ${ }^{29}$ The sudden change in mealtime during Ramadan may explain the nocturnal decrease in plasma leptin levels. The difference between the findings of the two studies could be explained by the fact that the second study controlled for lifestyle changes and environmental conditions, while the first study did not, which might have resulted in a delay in the circadian rhythm of leptin in the first study.

So far, only one study has evaluated circadian rhythm of ghrelin during Ramadan diurnal IF while controlling for eating habits and environmental conditions. ${ }^{29} \mathrm{~A}$ cosinor analysis revealed no significant changes in the "acrophase" of the circadian rhythm of ghrelin. ${ }^{29}$

In summary, the above-reviewed studies suggest that during Ramadan diurnal IF, there is a delay in the circadian clock. However, when eating habits and environmental conditions are accounted for, diurnal IF does not significantly affect the circadian rhythm of body temperature and melatonin.

\section{Effects of diurnal IF on sleep Subjective assessment of sleep}

When assessing the influences of diurnal IF during Ramadan on sleep, it is essential to consider the accompanying lifestyle changes during the holy month. For example, in some Muslim countries, the beginning of work is delayed and work duration is shortened during Ramadan. Some of the earlier studies that assessed sleep patterns during Ramadan used sleep diaries and self-reported data. Studies that assessed sleep/wake pattern in three Muslim countries revealed a significant sudden delay in bedtime and wake time during Ramadan in performers of diurnal IF. ${ }^{21,23,30,39,40}$

Interestingly, one study demonstrated bedtime delay during Ramadan even in nonfasting subjects. ${ }^{23}$ This documented delay in bedtime and rise time reflects the delay in the start of work during Ramadan. 


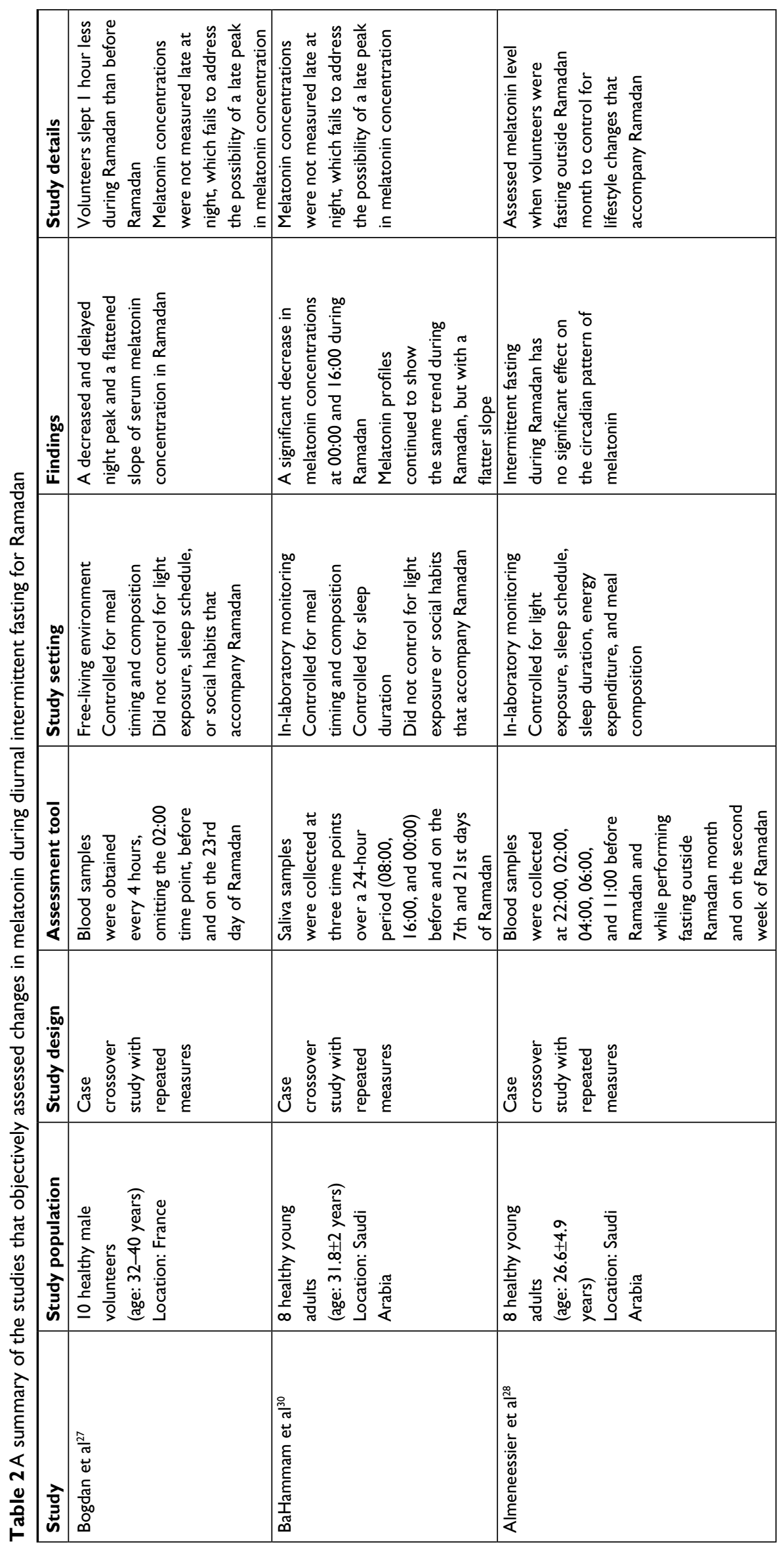


With regard to sleep duration, conflicting results were reported during Ramadan. Whereas some studies reported a reduction of sleep duration during Ramadan, ${ }^{39-41}$ others reported no significant alterations in nighttime sleep duration during Ramadan. ${ }^{21,23}$ Nevertheless, in a study that assessed sleep duration objectively using actigraphy in eight fasting (diurnal IF) and eight nonfasting volunteers in a free-living environment during Ramadan, bedtime and wake time were delayed in the fasting volunteers, and a significant reduction in total sleep time was observed in the fasting group. ${ }^{42}$

The discrepancies between the above studies may reflect cultural and lifestyle variations among countries or the use of different subjective assessment tools. ${ }^{12,13}$

\section{Objective assessment of sleep Sleep architecture}

A few studies have objectively measured sleep parameters during the month of Ramadan among volunteers practicing diurnal IF using in-laboratory polysomnography and homebased ambulatory unattended polysomnography. ${ }^{25,30,43,44}$ Table 3 summarizes the studies that have evaluated sleep architecture using polysomnography during Ramadan diurnal IF. In studies that controlled for sleep/wake pattern, diurnal IF had no effect on nonrapid eye movement sleep stages, stage shifts, arousal index, or measured cardiorespiratory parameters..$^{25,30,44}$ One study objectively assessed sleep among young athletes and revealed no changes in total sleep time during Ramadan diurnal IF; however, the study reported a significant increase in the number of awakenings. ${ }^{43}$ Nevertheless, a major limitation of the last study is the fact that the study athletes suddenly changed their primary sleep time from night to daytime, and the study did not account for naps. Therefore, the abrupt changes in bedtime and rise time may have accounted for the observed changes in sleep architecture. ${ }^{17,45}$

A recent study controlled for the lifestyle changes associated with Ramadan, such as sleep/wake pattern, caloric consumption, meal composition, and light exposure to eliminate variables other than meal timing that might affect sleep architecture; it objectively assessed sleep architecture at baseline, during diurnal IF outside Ramadan, during Ramadan, and during a nonfasting period after Ramadan. ${ }^{44}$ Rapid eye movement (REM) sleep proportion decreased while fasting during and outside Ramadan compared with the baseline; however, the proportion of REM sleep reverted to normal during recovery (after Ramadan) ${ }^{44}$ Three other studies reported a reduction in REM sleep during Ramadan. ${ }^{25,30,43}$ In agreement with the above, studies that assessed the effect of experimental fasting on animals reported similar changes in sleep architecture..$^{46}$ REM sleep disappeared in piglets following 18 hours of fasting, which reverted to normal after feeding. ${ }^{46}$

Several hypotheses have been proposed to account for the decrease in REM sleep during fasting; however, the precise mechanisms are unknown. ${ }^{12}$ In addition, the clinical consequences of reduced REM sleep during fasting are unknown. Proposed theories to understand the decrease in REM sleep during fasting include the documented nocturnal rise in cortisol and insulin, ${ }^{47,48}$ and the possible increase in nocturnal body temperature as a result of eating exclusively at night during diurnal IF. ${ }^{25,26}$ Increased nocturnal temperature could result in a reduction in REM sleep proportion. ${ }^{49,50}$ Another proposed mechanism is the interruption of sleep in the early morning (predawn) for the predawn meal (Suhur), which is the period in which the major fraction of REM sleep usually occurs. ${ }^{30}$

\section{Sleep latency}

With regard to sleep latency during Ramadan IF, studies have reported conflicting results. Roky et al showed a significant increase in sleep latency and a significant reduction in nocturnal sleep time.$^{25}$ However, in that study, dinner was served 30 minutes before going to bed; the short time between dinner and bedtime could have affected sleep latency. Later studies that provided dinner at an earlier time (3-3.5 hours before bedtime) and controlled daytime naps revealed no change in sleep latency. ${ }^{13,30,44}$

The above-reviewed studies suggest that when dinner is provided early and daytime naps are controlled for, diurnal IF does not have major influence in sleep architecture. The only change in sleep architecture consistently reported is the reduction in REM sleep. ${ }^{44}$ However, in a free-living environment that does not control for confounders that usually accompany Ramadan such as mealtimes or the daytime naps, sleep latency and sleep architecture may be disturbed. ${ }^{43}$

\section{Daytime sleepiness}

A discussion of the effect of diurnal IF on sleep would not be complete without reviewing the impact of daytime fasting on daytime sleepiness. Several studies have assessed the interaction between daytime sleepiness and diurnal IF during Ramadan both subjectively ${ }^{21,23,30,39,40,42,44,51}$ and objectively. ${ }^{30,44,51}$ However, these studies reported conflicting results, which may reflect the differences in the used assessment tools, the lack of objective assessment or the failure to control for potential confounders that could impact daytime 


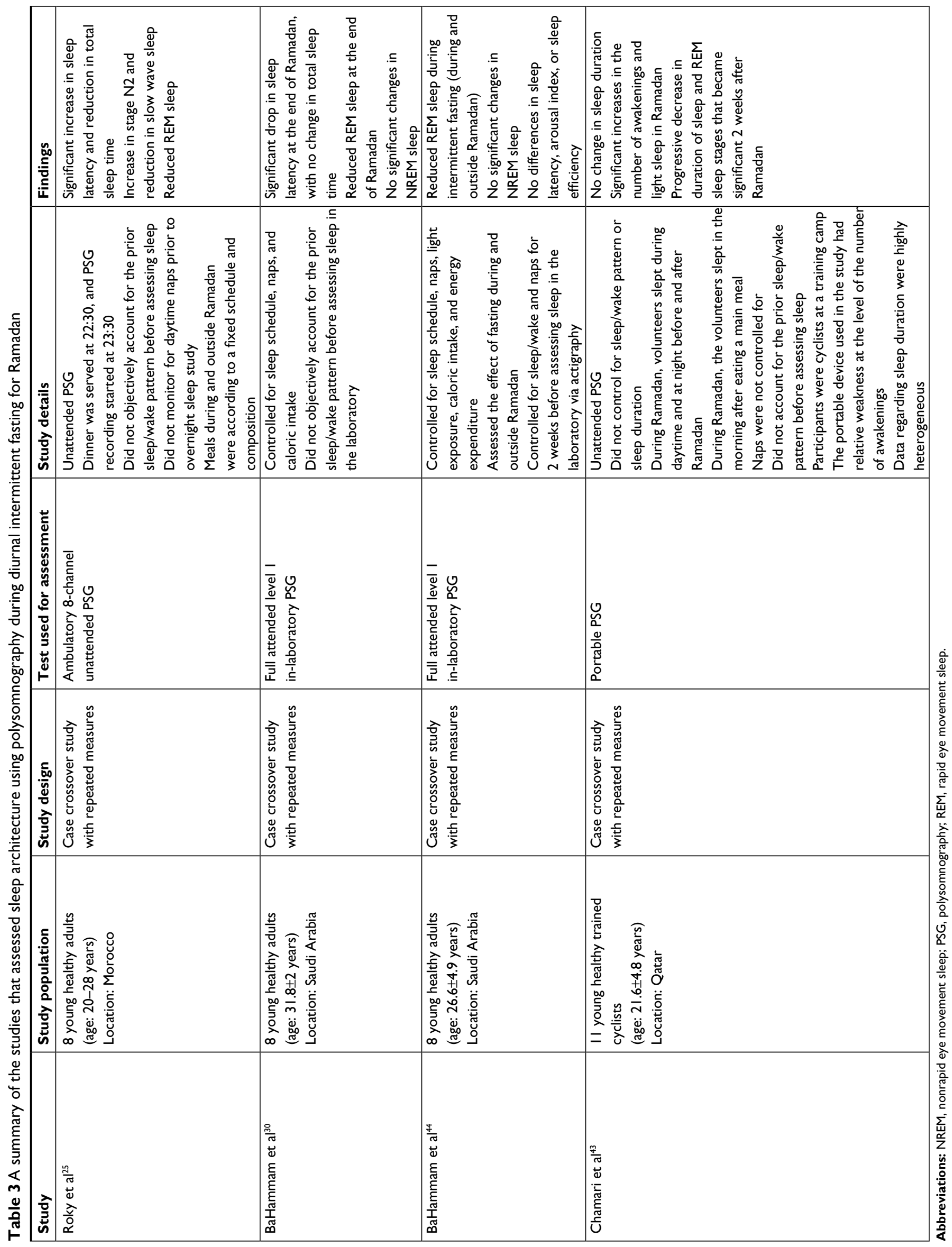


alertness. Table 4 summarizes the studies that have assessed daytime sleepiness during diurnal IF in Ramadan.

\section{Subjective assessment}

Studies that used the Epworth Sleepiness Scale (ESS) score to assess daytime sleepiness reported conflicting results. Some studies reported a significant increase in daytime sleepiness, ${ }^{21,40}$ whereas other studies demonstrated no significant

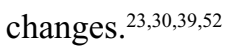

\section{Objective assessment}

In three studies, daytime sleepiness was measured objectively using the multiple sleep latency test (MSLT) under controlled conditions. ${ }^{30,44,51}$ The first study demonstrated an increase in daytime sleepiness at the 10:00 and 12:00 naps in the last week of Ramadan. ${ }^{51}$ However, the study used an unattended polysomnography recording device, which necessitated the end of the test 20 minutes after the beginning of recording regardless of sleep onset.

To avert the above shortcomings, a subsequent study used a standard attended MSLT. ${ }^{30}$ This study measured sleep latency, sleep onset latency, or wake efficiency on the first and third weeks of Ramadan and reported no changes compared with baseline measurements. ${ }^{30}$ Furthermore, spectral analysis of electroencephalography waves during each nap revealed no difference between Ramadan and baseline. ${ }^{30}$ However, the previous studies did not control for the possibility of sleep deprivation on the nights prior to assessing sleep and daytime sleepiness. A recent study aimed to objectively assess the impact of diurnal IF during Ramadan on daytime sleepiness in eight healthy subjects while providing a fixed sleep-wake schedule, fixed caloric intake, controlled light exposure, and objective monitoring of sleep duration for several nights before the assessment of daytime sleepiness. ${ }^{44}$ The assessment was performed on four occasions using attended MSLT and the ESS. During the first visit (adaptation night), wrist actigraphy was provided before the start of the study to monitor sleep pattern while at home to ensure that they had a fixed sleep-wake pattern. In subsequent visits, daytime sleepiness was measured using MSLT and ESS in the following order: 21 days before Ramadan after 1 week of diurnal IF "baseline fasting," 7 days before Ramadan "nonfasting baseline," on the 24th day of Ramadan, and 14 days after Ramadan "recovery." The study revealed no changes in the ESS across the four study times. In addition, the MSLT measurements revealed no differences in sleep latency among the "nonfasting baseline," "baseline fasting," "Ramadan," and "recovery" time points.
Another study assessed the impact of diurnal IF during Ramadan on drowsiness under controlled conditions with a fixed light/dark exposure, caloric intake, sleep/wake schedule and sleep duration via measuring total blink duration (measured using infrared reflectance (IR) oculography) and the mean reaction time (MRT) ${ }^{61}$ The results of this study showed that diurnal intermittent fasting had no impact on drowsiness or vigilance. ${ }^{61}$

$\mathrm{CR}$ has been reported to enhance alertness and to upregulate orexin gene expression in animal models. ${ }^{53,54}$ It has been proposed that mammals, when exposed to a negative energy balance, respond with enhanced alertness to improve their capability to find food. ${ }^{55,56}$ It has also been shown in animals that orexin (an excitatory neurotransmitter) neurons are important for this adaptive response during fasting. . $^{56,57}$ Moreover, orexin neurons interact closely with the "hypothalamic-pituitary-adrenal" (HPA) axis, ${ }^{58}$ and stimulation of the sympathetic and HPA tone secondary to fasting may further induce arousal through the orexin system..$^{59}$ Therefore, a recent study assessed the effects of diurnal IF during the month of Ramadan on plasma orexin while controlling for lifestyle changes that may accompany Ramadan, like sleep duration, bedtime and wake time, energy expenditure, light exposure, and food, in eight healthy young men. ${ }^{60}$ The results showed that plasma orexin levels increased during the fasting hours.

\section{Summary}

The impact of diurnal IF on circadian rhythm, sleep, and daytime sleepiness is an exciting topic that deserves future research. Earlier studies have suggested that diurnal IF during Ramadan causes a delay in the circadian rhythm of core body temperature and hormonal secretion. However, more recent studies controlling for lifestyle changes that may influence circadian rhythm showed no effects of diurnal IF on the circadian rhythm.

In free-living, unconstrained environments that do not control for lifestyle changes, studies have revealed abrupt significant delays in bedtime and rise time. Nevertheless, studies that controlled for environmental conditions and sleep-wake pattern revealed no significant changes in sleep architecture, indicating that the earlier reported changes could be secondary to concomitant lifestyle changes rather than the fasting act per se. The only consistent change in all studies that assessed sleep architecture during diurnal IF is a decrease in the amount of REM sleep.

Although earlier subjective studies reported an increase in daytime sleepiness during diurnal IF, subsequent studies that controlled for confounding factors reported no changes. 


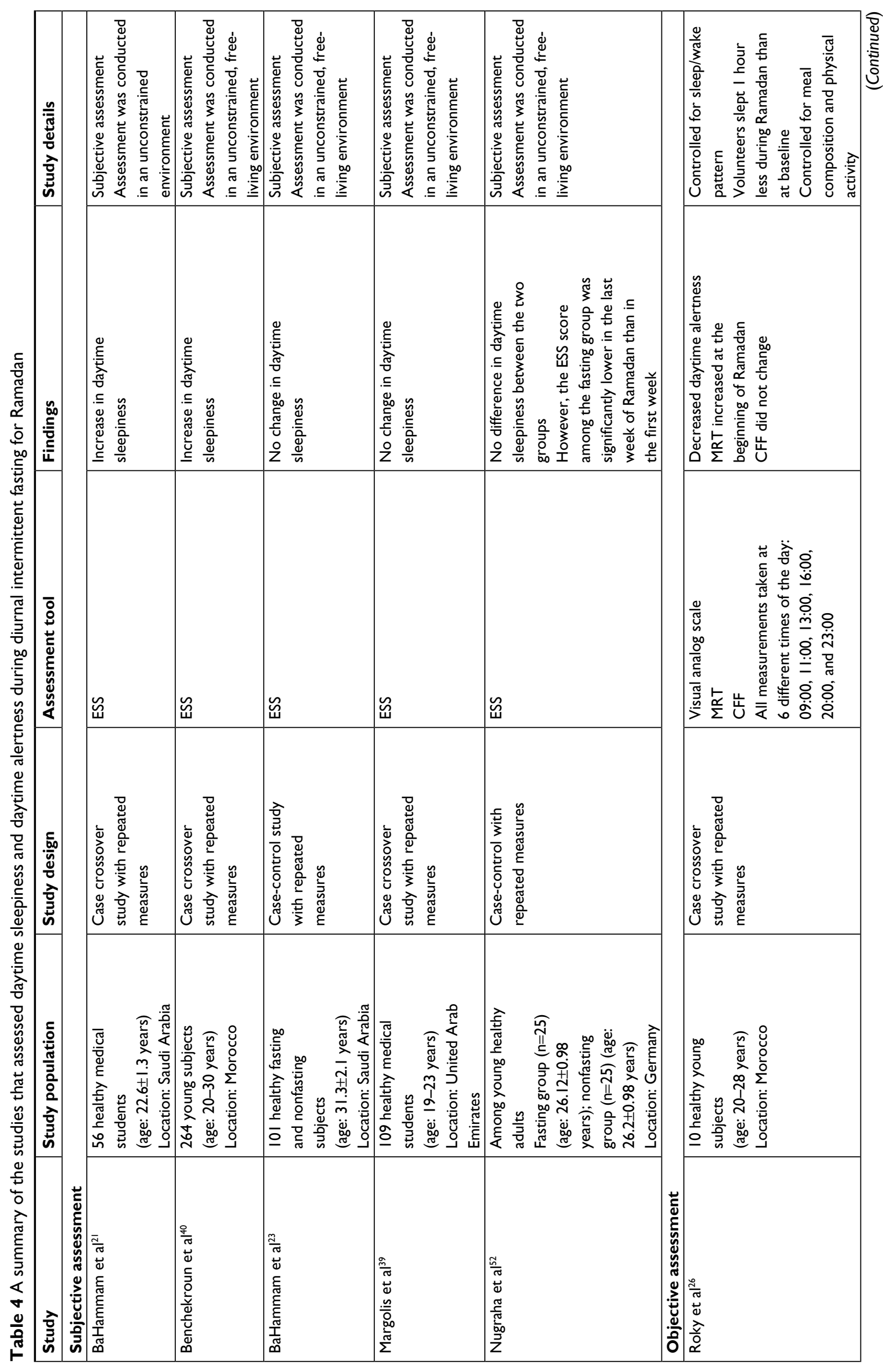




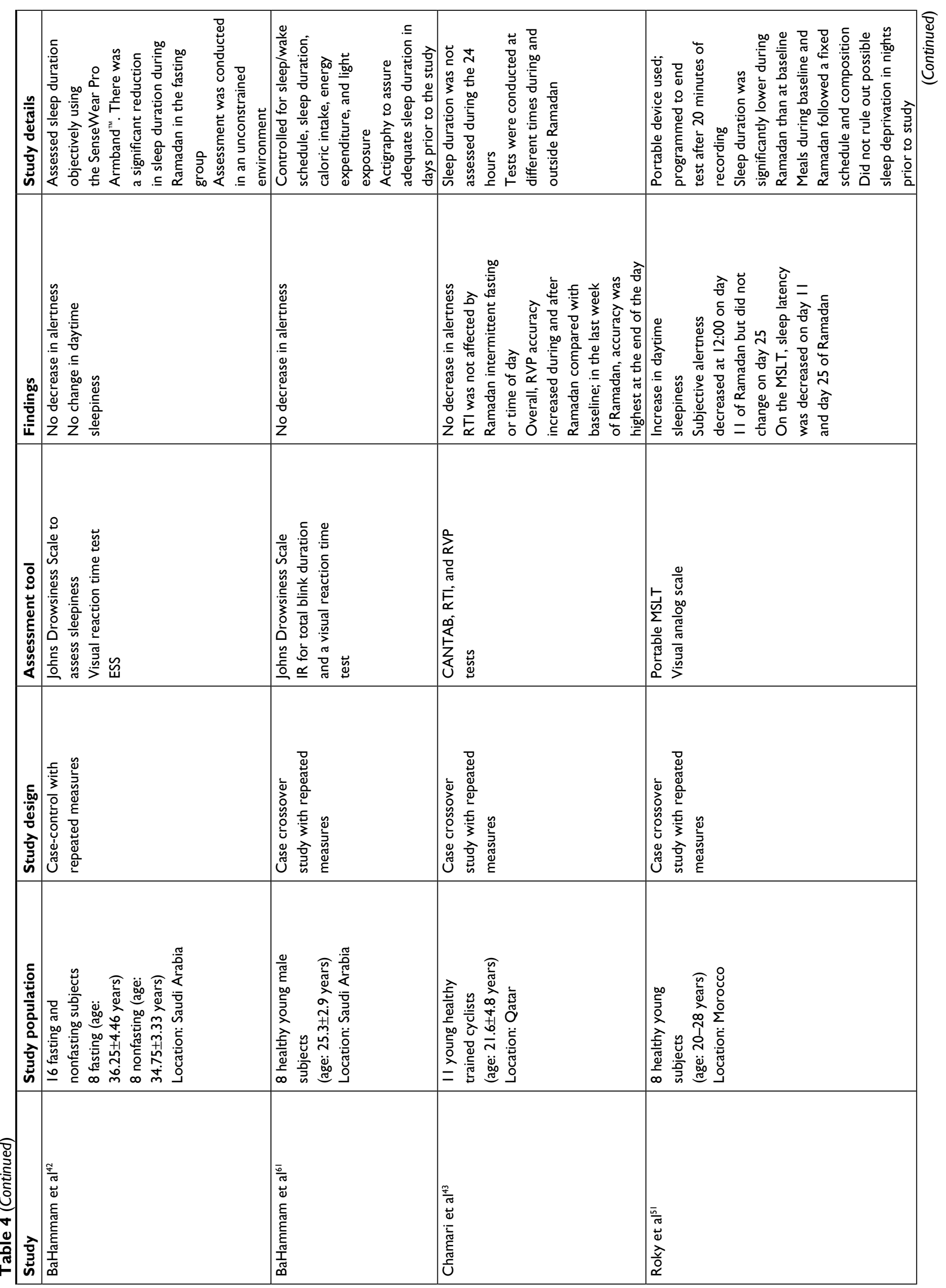




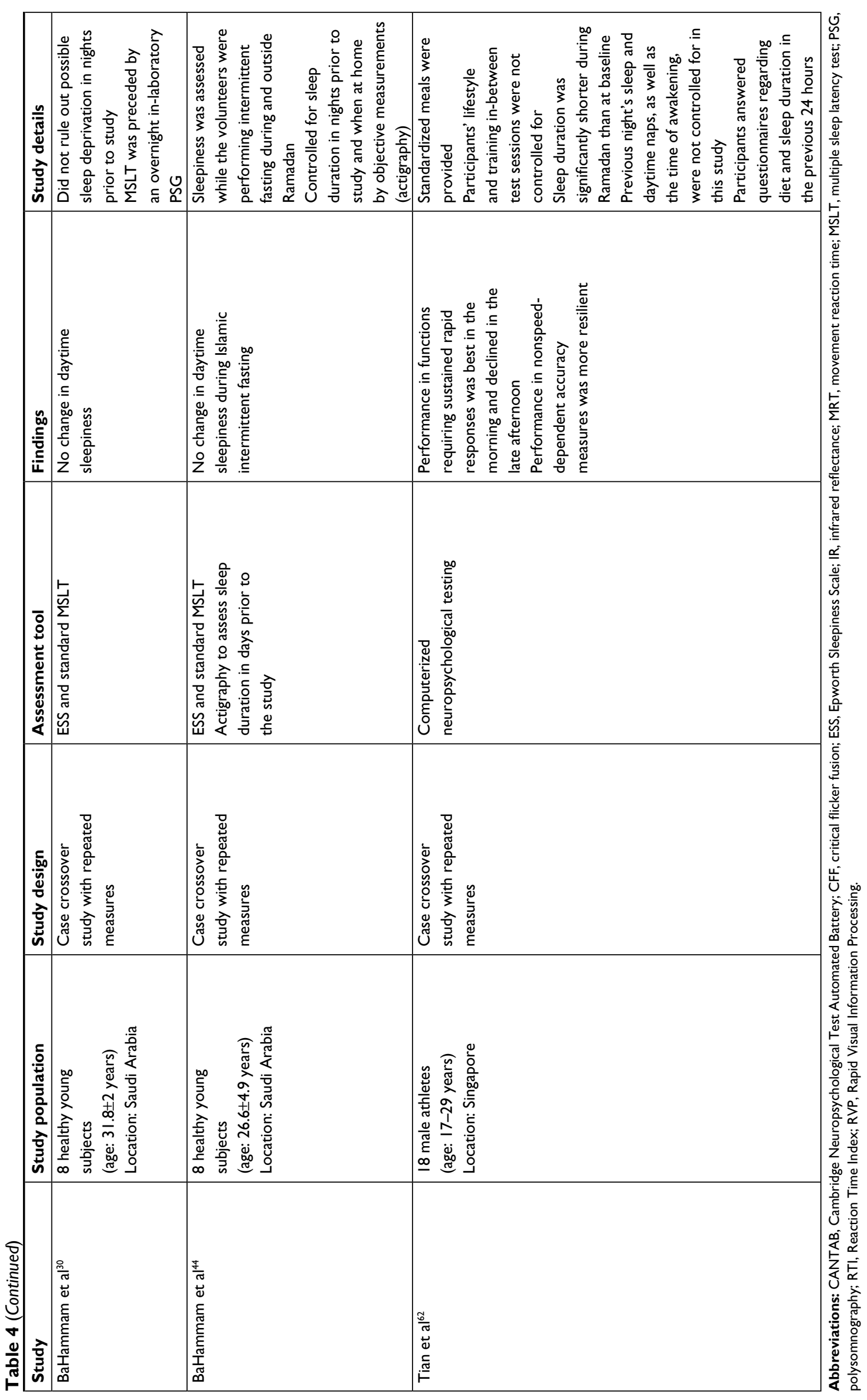


Larger studies that control for potential confounding factors, such as environmental and cultural conditions (the delay in the start of work and school, light exposure, exercise, and meal composition), are warranted to assess the impact of diurnal IF on circadian rhythms, sleep, and daytime sleepiness.

\section{Acknowledgments}

This project was funded by the Strategic Technologies Program of the National Plan for Sciences and Technology and Innovation in the Kingdom of Saudi Arabia (MED511-02-08).

\section{Disclosure}

The authors report no conflicts of interest in this work.

\section{References}

1. Almeneessier AS, Pandi-Perumal SR, Bahammam AS. Intermittent fasting, insufficient sleep, and circadian rhythm: interaction and effects on the cardiometabolic system. Curr Sleep Med Rep. 2018;4(3):179-195.

2. Wehrens SMT, Christou S, Isherwood C, et al. Meal timing regulates the human circadian system. Curr Biol. 2017;27(12):e1763:1768-1775.

3. Patterson RE, Sears DD. Metabolic effects of intermittent fasting. Annu Rev Nutr. 2017;37:371-393.

4. Schwartz DL. Fasting. In: Bagnall RS, Brodersen K, Champion CB, Erskine A, Huebner SR, editors. The Encyclopedia of Ancient History. 1st ed. Boston: Blackwell Publishing Ltd; 2013:2644-2645.

5. Giorda MC, Bossi L, Messina E. Food and Religion (in Public Food Service). 1st ed. Turin, Italy: Consorzio Risteco; 2014. Available from: http://www.eurel.info/IMG/pdf/report_2014_food_and_religion_in_ public_food_service_.pdf. Accessed September 30, 2018.

6. Longo VD, Mattson MP. Fasting: molecular mechanisms and clinical applications. Cell Metab. 2014;19(2):181-192.

7. Anson RM, Guo Z, de Cabo R, et al. Intermittent fasting dissociates beneficial effects of dietary restriction on glucose metabolism and neuronal resistance to injury from calorie intake. Proc Natl Acad Sci US A. 2003;100(10):6216-6220.

8. Varady KA, Dam VT, Klempel MC, et al. Effects of weight loss via high fat vs. low fat alternate day fasting diets on free fatty acid profiles. Sci Rep. 2015;5:7561.

9. Harvie MN, Pegington M, Mattson MP, et al. The effects of intermittent or continuous energy restriction on weight loss and metabolic disease risk markers: a randomized trial in young overweight women. Int $J$ Obes. 2011;35(5):714-727.

10. Chaix A, Zarrinpar A, Miu P, Panda S. Time-restricted feeding is a preventative and therapeutic intervention against diverse nutritional challenges. Cell Metab. 2014;20(6):991-1005.

11. Almeneessier AS, Pandi-Perumal SR, Bahammam AS. Intermittent fasting, insufficient sleep, and circadian rhythm: interaction and effects on the cardiometabolic system. Curr Sleep Med Rep. 2018;4(3):179-195.

12. Qasrawi SO, Pandi-Perumal SR, Bahammam AS. The effect of intermittent fasting during Ramadan on sleep, sleepiness, cognitive function, and circadian rhythm. Sleep Breath. 2017;21(3):577-586.

13. Bahammam A. Does Ramadan fasting affect sleep? Int J Clin Pract. 2006;60(12):1631-1637.

14. Van Laake LW, Lüscher TF, Young ME. The circadian clock in cardiovascular regulation and disease: lessons from the Nobel Prize in Physiology or Medicine 2017. Eur Heart J. 2018;39(24):2326-2329.

15. Zelinski EL, Deibel SH, Mcdonald RJ. The trouble with circadian clock dysfunction: multiple deleterious effects on the brain and body. Neurosci Biobehav Rev. 2014;40:80-101.
16. St-Onge MP, Ard J, Baskin ML, et al. Meal timing and frequency: implications for cardiovascular disease prevention: a scientific statement from the American Heart Association. Circulation. 2017;135(9): e96-e121.

17. Oosterman JE, Kalsbeek A, La Fleur SE, Belsham DD. Impact of nutrients on circadian rhythmicity. Am J Physiol Regul Integr Comp Physiol. 2015;308(5):R337-R350.

18. De Martino MM. The architecture of day sleeping and the sleepwake cycle in nurses in their working shifts. Rev Esc Enferm USP. 2009;43(1):194-199.

19. Mongrain V, Carrier J, Dumont M. Chronotype and sex effects on sleep architecture and quantitative sleep EEG in healthy young adults. Sleep. 2005;28(7):819-827.

20. Patton DF, Mistlberger RE. Circadian adaptations to meal timing: neuroendocrine mechanisms. Front Neurosci. 2013;7:185.

21. Bahammam A. Sleep pattern, daytime sleepiness, and eating habits during the month of Ramadan. Sleep Hypnosis. 2003;5:165-174.

22. Horne JA, Ostberg O. A self-assessment questionnaire to determine morningness-eveningness in human circadian rhythms. Int J Chronobiol. 1976;4(2):97-110.

23. Bahammam A. Assessment of sleep patterns, daytime sleepiness, and chronotype during Ramadan in fasting and nonfasting individuals. Saudi Med J. 2005;26(4):616-622.

24. Bahammam A, Alrajeh M, Albabtain M, Bahammam S, Sharif M. Circadian pattern of sleep, energy expenditure, and body temperature of young healthy men during the intermittent fasting of Ramadan. Appetite. 2010;54(2):426-429.

25. Roky R, Chapotot F, Hakkou F, Benchekroun MT, Buguet A. Sleep during Ramadan intermittent fasting. J Sleep Res. 2001;10(4):319-327.

26. Roky R, Iraki L, Hajkhlifa R, Lakhdar Ghazal N, Hakkou F, Alertness D. Daytime alertness, mood, psychomotor performances, and oral temperature during Ramadan intermittent fasting. Ann Nutr Metab. 2000;44(3):101-107.

27. Bogdan A, Bouchareb B, Touitou Y. Ramadan fasting alters endocrine and neuroendocrine circadian patterns. Meal-time as a synchronizer in humans? Life Sci. 2001;68(14):1607-1615.

28. Almeneessier AS, Bahammam AS, Sharif MM, et al. The influence of intermittent fasting on the circadian pattern of melatonin while controlling for caloric intake, energy expenditure, light exposure, and sleep schedules: a preliminary report. Ann Thorac Med. 2017;12(3):183-190.

29. Alzoghaibi MA, Pandi-Perumal SR, Sharif MM, Bahammam AS. Diurnal intermittent fasting during Ramadan: the effects on leptin and ghrelin levels. PLoS One. 2014;9(3):e92214.

30. Bahammam A. Effect of fasting during Ramadan on sleep architecture, daytime sleepiness and sleep pattern. Sleep Biol Rhythms. 2004;2(2):135-143.

31. Miles ATR. Melatonin - A Diagnostic Marker in Laboratory Medicine? Oxford, UK: Oxford University Press; 1988.

32. Arendt J. Melatonin and the Mammalian Pineal Gland. London, UK: Chapman \& Hall; 1995.

33. Al-Hadramy MS, Zawawi TH, Abdelwahab SM. Altered cortisol levels in relation to Ramadan. Eur J Clin Nutr. 1988;42(4):359-362.

34. Sliman AF. Effect of fasting on some blood hormones in healthy Muslim males. Mu'tah J Res Stud. 1993;8:91-109.

35. Chik CL, Ho AK, Brown GM. Effect of food restriction on 24-h serum and pineal melatonin content in male rats. Acta Endocrinol. 1987;115(4):507-513.

36. Röjdmark S, Wetterberg L. Short-term fasting inhibits the nocturnal melatonin secretion in healthy man. Clin Endocrinol. 1989;30(4):451-457.

37. Azizi F. Research in Islamic fasting and health. Ann Saudi Med. 2002;22(3-4):186-191.

38. Bogdan A, Bouchareb B, TouitouY. Response of circulating leptin to Ramadan daytime fasting: a circadian study. Br J Nutr. 2005;93(4):515-518.

39. Margolis SA, Reed RL. Effect of religious practices of Ramadan on sleep and perceived sleepiness of medical students. Teach Learn Med. 2004;16(2):145-149. 
40. Taoudi Benchekroun M, Roky R, Toufiq J, Benaji B, Hakkou F. Epidemiological study: chronotype and daytime sleepiness before and during Ramadan. Therapie. 1999;54(5):567-572.

41. Lee JY, Tan CSS, Lee SWH, Css T, Swh L. Ramadan fasting alters sleep behavior in type 2 diabetes patients. J Diabetes. 2018.

42. Bahammam AS, Alaseem AM, Alzakri AA, Sharif MM. The effects of Ramadan fasting on sleep patterns and daytime sleepiness: an objective assessment. J Res Med Sci. 2013;18(2):127-131.

43. Chamari K, Briki W, Farooq A, Patrick T, Belfekih T, Herrera CP. Impact of Ramadan intermittent fasting on cognitive function in trained cyclists: a pilot study. Biol Sport. 2016;33(1):49-56.

44. Bahammam AS, Almushailhi K, Pandi-Perumal SR, Sharif MM. Intermittent fasting during Ramadan: does it affect sleep? J Sleep Res. 2014;23(1):35-43.

45. Fond G, Macgregor A, Leboyer M, Michalsen A. Fasting in mood disorders: neurobiology and effectiveness. A review of the literature. Psychiatry Res. 2013;209(3):253-258.

46. Oike H, Oishi K, Kobori M, Nutrients KM. Nutrients, clock genes, and chrononutrition. Curr Nutr Rep. 2014;3:204-212.

47. Sangiah S, Caldwell DF. Reduction of rapid eye movement (REM) sleep by glucose alone or glucose and insulin in rats. Life Sci. 1988;42(15):1425-1429.

48. Born J, Späth-Schwalbe E, Schwakenhofer H, Kern W, Fehm HL. Influences of corticotropin-releasing hormone, adrenocorticotropin, and cortisol on sleep in normal man. J Clin Endocrinol Metab. 1989;68(5):904-911.

49. Jouvet M, Buda C, Debilly G, Dittmar A, Sastre JP. Central temperature is the principal factor of regulation of paradoxical sleep in pontile cats. C R Acad Sci III. 1988;306(2):69-73.

50. Krieger DT, Glick SM. Sleep EEG stages and plasma growth hormone concentration in states of endogenous and exogenous hypercortisolemia or ACTH elevation. J Clin Endocrinol Metab. 1974;39(6):986-1000.

51. Roky R, Chapotot F, Benchekroun MT, et al. Daytime sleepiness during Ramadan intermittent fasting: polysomnographic and quantitative waking EEG study. J Sleep Res. 2003;12(2):95-101.
52. Nugraha B, Ghashang SK, Hamdan I, Gutenbrunner C. Effect of Ramadan fasting on fatigue, mood, sleepiness, and health-related quality of life of healthy young men in summer time in Germany: a prospective controlled study. Appetite. 2017;111:38-45.

53. González JA, Jensen LT, Iordanidou P, Strom M, Fugger L, Burdakov D. Inhibitory interplay between orexin neurons and eatingnhibitory interplay between orexin neurons and eating. Curr Biol. 2016;26(18):2486-2491.

54. Gao XB, Horvath TL. Feeding behavior: hypocretin/orexin neurons act between food seeking and eating. Curr Biol. 2016;26(18):R845-R847.

55. Mieda M. The roles of orexins in sleep/wake regulation. Neurosci Res. 2017;118:56-65.

56. Barson JR. Orexin/hypocretin and dysregulated eating: Promotion of foraging behavior. Brain Res. 2018.

57. Yamanaka A, Beuckmann CT, Willie JT, et al. Hypothalamic orexin neurons regulate arousal according to energy balance in mice. Neuron. 2003;38(5):701-713.

58. Winsky-Sommerer R, Yamanaka A, Diano S, et al. Interaction between the corticotropin-releasing factor system and hypocretins (orexins): a novel circuit mediating stress response. J Neurosci. 2004;24(50):11439-11448.

59. Schmeichel BE, Herman MA, Roberto M, Koob GF. Hypocretin neurotransmission within the central amygdala mediates escalated cocaine self-administration and stress-induced reinstatement in rats. Biol Psychiatry. 2017;81(7):606-615.

60. Almeneessier AS, Alzoghaibi M, Bahammam AA, et al. The effects of diurnal intermittent fasting on the wake-promoting neurotransmitter orexin-A. Ann Thorac Med. 2018;13(1):48-54.

61. Bahammam AS, Nashwan S, Hammad O, Sharif MM, Pandi-Perumal SR. Objective assessment of drowsiness and reaction time during intermittent Ramadan fasting in young men: a case-crossover study. Behav Brain Funct. 2013;9(1):32.

62. Tian HH, Aziz AR, Png W, Wahid MF, Yeo D, Constance Png AL. Effects of fasting during ramadan month on cognitive function in muslim athletes. Asian J Sports Med. 2011;2(3):145-153.
Nature and Science of Sleep

\section{Publish your work in this journal}

Nature and Science of Sleep is an international, peer-reviewed, open access journal covering all aspects of sleep science and sleep medicine, including the neurophysiology and functions of sleep, the genetics of sleep, sleep and society, biological rhythms, dreaming, sleep disorders and therapy, and strategies to optimize healthy sleep. The manuscript

\section{Dovepress}

management system is completely online and includes a very quick and fair peer-review system, which is all easy to use. Visit http://www. dovepress.com/testimonials.php to read real quotes from published authors. 